\title{
La televisión y su relación con el estado nutricional y frecuencia de consumo en niños de un conjunto habitacional de Talca, Chile
}

\author{
Television watching and its relationship with \\ food frequency and nutritional status in children \\ from a community of Talca, Chile
}

\begin{abstract}
Background: Television (TV) is a medium that massively delivers entertainment, information and culture in Chilean society contributing to create sedentary habits in children. Objective: To associate the time dedicated to TV watching with nutritional status and food habits in 3 to 6 years old children. Design: A cross-sectional study of 45 children residents of the population "Jardín del Este" in Talca was performed through home visits. Nutritional status was assessed and the time spent watching TV was recorded. Data was analyzed using Stata 10.1. Results: The majority (73.3\%) of children watched TV between 4 and 7 hours daily. The time devoted to watching TV was $3.47 \pm 1.0$ hours in eutrofic children, $4.7 \pm 0.9$ hours in overweight and $5.6 \pm 0.9$ hours in obese $(p<0.05)$. Children who consumed more servings of sugar daily spent on average more hours watching TV $(p<0,05)$. Conclusions: The time dedicated to watch TV at home influences the nutritional status and dietary habits of children.
\end{abstract}

Key words: television; nutritional status; food habits; physical activity.

\section{INTRODUCCIÓN}

La televisión (TV) es el medio de comunicación por excelencia, la cual crea y se involucra en los niveles micro y macro sociológicos que conforman el mundo actual, dada la gran cantidad de información y facilidad de consumo que ofrece este medio, puesto que en ella se reúnen voz e imagen a la vez (1).

Los cambios tecnológicos que se han producido en la industria televisiva durante las últimas décadas han sido fundamentales en la cual se incluyen la TV por cable, vía satélite y la digitalización de las plataformas de transmisión existentes (2). Prácticamente la totalidad de los hogares chilenos cuenta con al menos un aparato de televisión, y en especial los menores de edad, el tiempo que las personas dedican pasar frente al medio de comunicación, constituye la actividad que más horas ocupan las familias chilenas, después de trabajar y dormir (3). Varios estudios han propuesto que el exceso de tiempo destinado a ver TV se asocia con una variedad de resultados adversos en niños y niñas de edad preescolar, escolar y ado-
Miguel Ángel López E. (1)

Iris del Pilar Llanos J. (2)

Jacqueline Macarena Díaz A. (3)

(1) Departamento de Enfermería. Facultad de Ciencias de la Salud y de los Alimentos. Universidad del Bío Bío. Chillán, Chile (2) Mutual de Seguridad, Agencia Chillán, Chile (3) Carrera de Nutrición y Dietética. Universidad del Mar, sede Centro Sur. Talca, Chile

Dirigir la correspondencia a: Prof. Nutr. Miguel Ángel López Espinoza, MSc Avda. Andrés Bello s/n Universidad del Bío Bío Chillán, Chile Fono: 7-664 6086

E-mail:miglopeze@gmail.com

Este trabajo fue recibido el 24 de Mayo de 2012 y aceptado para ser publicado el 20 de Septiembre de 2012.

lescentes, tales como violencia, comportamientos agresivos, síntomas psicosomáticos, sobrepeso y obesidad (4-6). Por esta razón, la Academia Americana de Pediatría recomienda que los niños pequeños vean TV no más de dos horas al día (7).

En Chile, la encuesta Adimark-Time Ibope y Mindshare (2007), reveló que niños y niñas de 4 años ven 4 horas de TV por día, y el 75\% tiene un televisor en su dormitorio. Según la Sexta Encuesta Nacional del Consejo Nacional de Televisión (2008), destacan que hay 2,4 televisores por hogar (8).

Ver TV es un indicador importante de inactividad física, el cual a su vez explica los factores de riesgo cardiovascular (5). En escolares de primer año básico de escuelas públicas, se presentó una prevalencia de obesidad para el año 2008 de un 20,4\% (6) y en el 2010 un 23\% (9), lo cual se ha atribuido a distintas prácticas de alimentación y mayor sedentarismo relacionados con el avance socioeconómico alcanzado por el país (10).

En Chile, la influencia de la TV no está suficientemente asumida y no se le ha dado aún la atención que se merece en la 
conducta infantil (8), incluida su actividad física y alimentación; atendiendo que ya en la población adulta, el 39,3\% presenta sobrepeso, el 25,1\% obesidad, el 35,3\% presenta síndrome metabólico, el 15,7\% declara consumir cinco ó más porciones al día de frutas y verduras, el 39,4\% una ó más porciones a la semana de pescados, y que el $88,6 \%$ es sedentario de tiempo libre (11).

El objetivo de esta investigación fue estudiar el tiempo destinado a ver TV y su relación con el estado nutricional y los hábitos alimentarios.

\section{MATERIAL Y MÉTODO}

Diseño no experimental de tipo transversal. Se estudiaron niños de edad entre 3 y 6 años del barrio Jardín del Este de la ciudad de Talca (Región del Maule, Chile). Este conjunto habitacional, fue escogido por conveniencia para este estudio, el cual está ubicado en el sector suroriente de la ciudad y está constituido por 108 casas para familias de estratos socioeconómicos medio-bajo y bajo.

Se verificó con un primer recorrido en cada casa la cantidad de niños y niñas que habitan y su filiación con el cabeza de familia y/o sostenedor de cada casa, correspondiendo a 98 casos menores de 18 años, de los cuales la cantidad de niños y niñas entre 3 y 6 años, fueron 45 .

El levantamiento de datos se realizó mediante una visita domiciliaria entre el lunes y el viernes en cada casa (unidad muestral) de los sujetos de estudio (unidad de análisis), quienes acrediten residencia habitual en la unidad muestral, el cual a su vez pertenezca a manzanas de la población Jardín del Este de Talca. Luego de solicitar el consentimiento informado a los padres y/o al tutor legal y la disposición a participar del niño, se registró el nivel educacional de los padres; fecha de nacimiento, sexo y actividad física reportada durante una semana de los menores de edad. Se pidió a los padres registrar el tiempo empleado por el niño o niña en ver TV durante la última semana, en una hoja de registro dejada por la encuestadora, retirándola a la semana siguiente.

Luego, se evaluó el estado nutricional a partir de la medición del peso con una balanza marca Detecto ${ }^{\circledR}$, con tara máxima de $150 \mathrm{~kg}$ y precisión de 0,1 kilos, la cual se instaló en un lugar fijo; la talla se midió con altímetro marca Seca, modelo 240, de $200 \mathrm{~cm}$, con precisión de $1 \mathrm{~mm}$, utilizando las técnicas descritas por Quinteros (12). La edad al momento de la encuesta se calculó a partir de la diferencia entre las fechas de medición y nacimiento del menor. Se calcularon los indicadores peso/talla (PT), peso/edad (P/E) y talla/edad (T/E), y se realizó la evaluación nutricional en base a las tablas de la Organización Mundial de la Salud (OMS) para evaluación antropométrica (13).

Se aplicó una encuesta de tendencia de consumo cuantificada (ETCC) para determinar la frecuencia de alimentos consumidos por el niño, la cual se aplicó a los padres o tutor a cargo de él. El listado de alimentos fue tomado de la versión publicada por Urteaga y Pinheiro (14), y luego sometida a adaptación y validación de contenido a partir del juicio de tres expertos, completando el listado definitivo y agrupándose según el modelo de la pirámide alimentaria para la población chilena (15). Se solicitó a los encuestados remontarse siete días atrás para indicar la frecuencia de consumo de cada alimento y las porciones habituales de consumo según medidas caseras. Se determinó la mediana del consumo/día por cada grupo de alimentos.

Los datos fueron analizados con Stata 10,1. Las variables intervalares y de razón fueron descritas usando la media aritmética y desviación estándar, previa comparación de normalidad a través de la prueba de Shapiro- Wilk; en ausencia de normalidad, se utilizó mediana y rango intercuartílico (P75-P25). En tablas de contingencia se aplicó la prueba de independencia $(x 2)$. Para comparar medias de dos y tres grupos se aplicaron las pruebas de t-Student y Anova, y en ausencia de normalidad, las de Mann-Witney y Kruskal-Wallis, respectivamente. Todo el análisis inferencial se realizó considerando un nivel de significación de 0,05.

\section{RESULTADOS}

Se estudiaron 45 niños de 3 a 6 años, los cuales presentaron una edad media de 4,3 $\pm 0,9$ años, siendo $44,4 \%$ de sexo femenino $(n=20)$. Presentaron un promedio de peso y talla de $18,4 \pm 3,4$ kilos y $102,5 \pm 6,7 \mathrm{~cm}$, respectivamente. La tabla 1 indica que el $40 \%$ fueron diagnosticados con sobrepeso y $17,78 \%$ se clasificaron como obesos. El $42,2 \%$ presentó un estado nutricional normal y no hubo niños con bajo peso. No se presentó diferencia estadísticamente significativa entre niños y niñas $(p=0,885)$.

TABLA 1

Distribución de los niños por sexo según estado nutricional y tiempo en horas destinadas a ver televisión ( $\mathrm{n}=45$ )

\begin{tabular}{|c|c|c|c|}
\hline Características & Hombres & Mujeres & TOTAL \\
\hline \multicolumn{4}{|l|}{ Estado nutricional † } \\
\hline Normal & 9 & 10 & $19(42,22)$ \\
\hline Sobrepeso & 8 & 10 & $18(40,00)$ \\
\hline Obeso & 3 & 5 & $8(17,78)$ \\
\hline \multicolumn{4}{|l|}{ Tiempo en TV †† } \\
\hline 2 horas & 2 & 1 & $3(6,67)$ \\
\hline 3 horas & 4 & 5 & $9(20,00)$ \\
\hline 4 horas & 4 & 8 & $12(26,67)$ \\
\hline 5 horas & 6 & 8 & $14(31,11)$ \\
\hline 6 horas & 3 & 2 & $5(11,11)$ \\
\hline 7 horas & 1 & 1 & $2(4,44)$ \\
\hline $\begin{array}{l}n \text { n (\%) } \\
+\quad x 2=0,222(2 \mathrm{gl}) ; p=0,895 \\
+\quad x 2=1,7273(5 \mathrm{gl}) ; p=0,885\end{array}$ & & & \\
\hline
\end{tabular}


El $31,1 \%$ de los niños estudiados destinan cinco horas del día en ver TV, el $26,7 \%$ cuatro horas, el $20 \%$ tres horas y el $6,7 \%$ dos horas. El 11,1 y el 4,4\% destinan seis y siete horas en observar TV, respectivamente. No hubo diferencias significativas en la cantidad de horas destinadas a ver TV según sexo $(p=0,885)$.

La tabla 2 muestra el tiempo destinado a ver TV según estadio nutricional, reportándose que los obesos ocupan 5,6 $\pm 0,9$ horas en ver TV, los con sobrepeso 4,7 $\pm 0,9$ horas y los clasificados con estado nutricional normal 3,5 $\pm 1,0$ horas, cuyas diferencias son estadísticamente significativa $(p<0,001)$. Al desagregar por sexo, las niñas con estado nutricional normal vieron 3,4 $\pm 1,2$ horas, con sobrepeso 4,9 $\pm 0,9$ horas y en obesas $5,7 \pm 1,2$ horas $(p<0,05)$. En el grupo de los niños, presentaron medias de 3,5 $\pm 0,9$ horas, 4,5 $\pm 0,85$ horas $y$ $5,6 \pm 0,9$ horas respectivamente, también con diferencias estadísticamente significativa $(p<0,001)$.

Los niños y niñas con estado nutricional normal, sobrepeso y obesidad con padres de nivel educacional medio, ven TV en promedio 3,3 $\pm 0,8$ horas, 4,7 $\pm 1,0$ horas y 5,5 $\pm 0,8$ horas, respectivamente, habiendo diferencias estadísticamente significativa $(p<0,001)$. El tiempo destinado a ver TV en niños y niñas con estados nutricionales normal, sobrepeso y obesidad cuyos padres tenían un nivel educacional superior, no fue estadísticamente diferente $(p=0,0568)$.

Respecto al nivel educacional de la madre, el tiempo medio ocupado para ver TV fue de 4,50 $\pm 1,29$ horas en niños con estado nutricional normal y 4,50 $\pm 0,71$ horas con sobrepeso, sin presentar diferencia estadísticamente significativa $(p=0,3805)$; para madres con estudios medios se presentaron $3,45 \pm 1,13$ horas en niños normales, 4,77 $\pm 0,93$ horas con sobrepeso y $5,50 \pm 0,84$ horas en niños obesos, con diferencia estadísticamente significativa $(p<0,001)$. Para madres con nivel educacional superior presentó medias de 3,5 \pm 0,6 horas,
$4,3 \pm 1,2$ horas y $6,0 \pm 1,41$ horas para estadios nutricionales normal, sobrepeso y obesidad respectivamente, sin presentar diferencia estadísticamente significativa $(p=0,0663)$.

Los niños y niñas que no realizaban actividad física con diagnóstico nutricional de normalidad, sobrepeso y obesidad, presentaron en promedio 4,0 $\pm 1,4,4,8 \pm 0,9$ y 5,6 6 0,9 horas de TV, sin diferencia estadísticamente significativa $(p=0,0810)$. Quienes presentaron actividad física con una frecuencia entre una y cinco veces a la semana, el tiempo medio destinado a ver TV fue de 3,5 $\pm 0,9$ horas en niños y niñas con estado nutricional normal, 4,8 $\pm 0,9$ horas en menores con sobrepeso y 5,6 \pm 0,9 horas en obesos, diferencia estadísticamente significativa $(p<0,001)$. En el grupo de niños y niñas que realizaron actividad física entre 6 y 10 veces por semana, no se presentaron diferencias estadísticamente significativas $(p=0,6850)$, respecto al tiempo destinado a ver TV en niños y niñas con estado nutricional normal $(3,5 \pm 1,3$ horas) y con sobrepeso $(4,0 \pm 1,4)$.

La tabla 3 señala que la media de porciones de verduras consumidas de niños y niñas que destinan entre una y tres horas a ver TV fue significativamente mayor en comparación a los que destinan más de 4 horas al día $(p<0,03)$, mientras que en el grupo de los azúcares el promedio de porciones fue estadísticamente mayor en los niños y niñas que veían cuatro horas o más respecto de los que veían entre una y tres horas $(p<0,05)$. No se presentaron diferencias estadísticamente significativas en el consumo de porciones/día para los grupos de cereales, frutas, lácteos, carnes, pescados y aceites de niños y niñas que ven TV entre una y tres horas respecto de los que ven entre cuatro horas ó más.

\section{DISCUSIÓN}

El propósito del estudio fue indagar si existía correlación entre el estado nutricional y los hábitos alimentarios, con el

\section{TABLA 2}

Tiempo destinado a ver televisión (horas/día) según sexo, nivel escolaridad del padre y madre por estado nutricional de los niños $(n=45)$

\begin{tabular}{|c|c|c|c|c|}
\hline \multirow[t]{2}{*}{ Tiempo TV (horas/día) } & \multicolumn{3}{|c|}{ Estado nutricional de los niños } & \multirow[t]{2}{*}{$\mathrm{p}$} \\
\hline & Normal & Sobrepeso & Obesidad & \\
\hline Total en horas $(n=45)+$ & $3,47 \pm 1,02$ & $4,67 \pm 0,91$ & $5,62 \pm 0,92$ & $<0,001+$ \\
\hline \multicolumn{5}{|l|}{ Sexo del niño: } \\
\hline Femenino + & $3,44 \pm 1,24$ & $4,88 \pm 0,99$ & $5,67 \pm 1,15$ & $0,0122+$ \\
\hline Masculino + & $3,50 \pm 0,85$ & $4,50 \pm 0,85$ & $5,60 \pm 0,89$ & $0,0007+$ \\
\hline \multicolumn{5}{|l|}{ Nivel educacional padre: } \\
\hline Básica + & $6,00 \pm 0,00$ & $5,00 \pm 0,00$ & $\mathrm{~s} / \mathrm{i}$ & * \\
\hline Media + & $3,33 \pm 0,82$ & $4,73 \pm 1,01$ & $5,50 \pm 0,84$ & $<0,001+$ \\
\hline Superior + & $3,33 \pm 1,15$ & $4,50 \pm 0,84$ & $6,00 \pm 1,41$ & $0,0568+$ \\
\hline \multicolumn{5}{|l|}{ Nivel educacional madre: } \\
\hline Básica + & $4,50 \pm 1,29$ & $4,50 \pm 0,71$ & $\mathrm{~s} / \mathrm{i}$ & $0,3805+$ \\
\hline Media + & $3,45 \pm 1,13$ & $4,77 \pm 0,93$ & $5,50 \pm 0,84$ & $0,0007+$ \\
\hline Superior + & $3,50 \pm 0,58$ & $4,33 \pm 1,15$ & $6,00 \pm 1,41$ & $0,0663+$ \\
\hline \multicolumn{5}{|l|}{ Actividad física: } \\
\hline No realiza actividad + & $4,00 \pm 1,41$ & $4,82 \pm 0,98$ & $5,57 \pm 0,98$ & $0,0810+$ \\
\hline Entre 1 y $5+$ & $3,00 \pm 1,00$ & $5,00 \pm 1,00$ & $6,00 \pm 0,00$ & 0,0098 t† \\
\hline Entre 6 y $10+$ & $3,50 \pm 1,29$ & $4,00 \pm 1,41$ & $s / i$ & $0,6850+$ \\
\hline
\end{tabular}


tiempo empleado en observar TV en un grupo de niños de 3 y 6 años, considerando que la obesidad infantil es un importante predictor de obesidad en la adultez, y por tanto, con mayor riesgo de enfermedades cardíacas, hipertensión, diabetes y cáncer (14). Los resultados del estudio indicaron que el $57,8 \%$ de los niños, presentaron malnutrición por exceso, de los cuales el $17,8 \%$ se clasificó como obesos, cifra menor a la comparada con las series publicadas de la Junta Nacional de Auxilio Escolar y Becas (JUNAEB), citada por Crovetto, 2011 (9). Independiente de lo anterior, este problema está presente en 1 de cada 5 casos aproximadamente, originada fundamentalmente por una ingesta calórica superior a la requerida, a partir de un alto consumo de hidratos de carbono en forma de cereales, de preferencia el pan (17), productos azucarados y la inclusión dentro de la dieta de productos denominados "comida rápida", lo que conlleva a un excesivo depósito de grasa en el organismo (18), en la cual depende de muchos factores, entre los cuales está el tiempo destinado a actividades sedentarias, tal como es la televisión.

Sólo un $6,7 \%$ de los niños que participaron del estudio vieron dos horas al día TV. Según un trabajo con menores de edad norteamericanos, pasan un promedio de 3 a 4 horas diarias viendo TV (19). En Chile, un estudio realizado en menores de dos años señala que el 35\% veía TV y el 37,5\% jugaba con un miembro de la casa; lo que indica que ya están iniciando vidas sedentarias sometidas a información y estímulos envasados no personalizados de la TV y que una proporción relativamente baja es estimulado por la familia, por lo que cabe plantearse si el estilo de vida de la familia en torno al menor se está delegando en la TV, como funciones que no le corresponden (20).

La disminución del tiempo destinando a la TV disminuye el índice de masa corporal (IMC), perímetro de cintura, y pliegue tricipital (6), mejorando la salud infantil; por esta razón, la Academia Americana de Pediatría recomienda limitar el tiempo en no más de 2 horas al día y que los niños menores de 2 años no la usen (9). Debido a que el hábito de ver TV se fija en los años preescolares, las intervenciones para reducir el tiempo destinado a ella deben comenzar a una edad temprana (21).

Los niños y niñas con sobrepeso y obesidad presentaron en promedio mayor tiempo viendo TV, en comparación con aquellos de estado normal $(p<0,01)$. El estudio Génesis (Grecia) indica que en una muestra representativa de 2.374 niños griegos entre 1 y 5 años la mayoría de los participantes $(74,0 \%)$ gastó menos de 2 horas al día viendo TV. Los niños que gastaron más de 2 horas diarias en ver TV tienen un mayor consumo de energía en comparación con niños que miraban TV menos de 2 horas diarias, luego de ajustar por posibles factores de confusión ( $p<0,001)(22)$. Un estudio de Loaiza y Atalah (2006), indica que los escolares entre 6 y 7 años que veían 4 o más horas de TV/día o que realizaban menos horas de actividad física tenían un riesgo 1,7 veces mayor de desarrollar obesidad $(p<0,05)(18)$.

Considerando que los resultados del estudio se basa en niños de familias insertas en un contexto socioeconómico medio-bajo y bajo, la TV se incorpora como el más probable único medio de entretenimiento del menor y su familia, puesto que adquiría mayor importancia cotidiana, debido a un contexto de mayor encierro y de menos dispositivos tecnológicos y comunicacionales (23).

Respecto a los hábitos alimentarios, los niños que consumían mayor cantidad de verduras dedicaban menos tiempo a la TV $(p<0,05)$; mientras que el mayor consumo de azúcar se concentró en niños que pasaban mayor cantidad de horas viendo TV $(p<0,05)$, hechos que han sido reportado por otros estudios $(18,22,27)$, especialmente en varones, quienes según nuestros resultados presentaron una mayor diferencia significativa, similar a lo planteado en un artículo publicado el año 2010 basado en niños europeos, el cual reveló que ver TV y el sexo masculino son importantes predictores para la comida rápida, bebidas azucaradas y peso corporal (26). Los análisis de contenido han demostrado que la alimentación es la categoría que con más frecuencia se anuncia en la TV de los niños, centrándose en productos muy dulces; más recientemente las promociones de comida rápida ha ido creciendo (27), en donde el tiempo de TV como actividad sedentaria, y su publicidad, se postula como un incentivador para el consumo de azúcar y grasas $(6,28)$.

No es sorprendente deducir que la televisión contribuye en los hábitos alimentarios poco saludables en niños y niñas (24), en donde la programación emitida, incluida la propaganda alimentaria bajo un ambiente virtual, idealiza el mensaje y fideliza al menor en cuanto a la posibilidad de elegir hora, canal y contenidos que desea ver de manera simple, rápida y cómoda. En este contexto, los padres juegan un papel importante en el fomento de una adecuada nutrición, en inculcar

TABLA 3

Tiempo destinado en ver televisión según porciones/semana de grupos de alimentos

\begin{tabular}{|c|c|c|c|}
\hline \multirow[t]{2}{*}{ Grupos de alimentos } & \multicolumn{2}{|c|}{ Tiempo de TV (horas/día) } & \multirow[t]{2}{*}{$p$} \\
\hline & $1-3$ horas & 4 ó más horas & \\
\hline Cereales + & $8,98 \pm 1,43$ & $9,03 \pm 1,48$ & $0,5400+$ \\
\hline Verduras + & $4,00 \pm 0,60$ & $3,39 \pm 0,93$ & $0,0214+$ \\
\hline Frutas + & $4,33 \pm 1,15$ & $4,03 \pm 1,16$ & $0,2209+$ \\
\hline Lácteos ++ & $7,00 \pm 0,00$ & $7,00 \pm 1,00$ & 0,1820 †十 \\
\hline Carnes + & $3,42 \pm 0,51$ & $3,45 \pm 0,62$ & $0,4252+$ \\
\hline Pescados ++ & $0,42 \pm 0,51$ & $0,45 \pm 0,51$ & $0,8231+\dagger$ \\
\hline Aceites + & $3,58 \pm 1,16$ & $3,48 \pm 0,91$ & $0,3833+$ \\
\hline Azúcares ++ & $4,67 \pm 3,45$ & $6,67 \pm 1,29$ & $0,0341+\dagger$ \\
\hline \multicolumn{4}{|c|}{$\begin{array}{l}+ \text { Media aritmética } \pm \text { Desviación típica }+ \text { Prueba de t-Student } \\
++ \text { Mediana } \pm \text { Rango intercuartílico }++ \text { Prueba de Mann-Whitney } \\
\text { Valor no calculado, casos insuficientes. } \\
\text { / } / \mathrm{i}=\text { Sin información }\end{array}$} \\
\hline
\end{tabular}


buenos hábitos alimentarios y garantizar alimentos saludables disponibles y accesibles en el hogar, lo cual implica que estén más atraídos a consumir alimentos nutritivos (25), además del ejercicio el acompañamiento y control de los contenidos ofrecidos por la televisión cuando se les exponga, puesto que el menor le es difícil distinguir la realidad-ficción de lo que recibe de la TV. Esto último es atendido por la maduración cerebral del niño y niña, puesto que en menores de 5 años, existe una marcada capacidad de fantasía, en la cual perciben las imágenes de la TV como reales y verdaderas y con capacidad de razonamiento crítico y relacional limitado (8). Ya el año 2007, el Reino Unido dio a conocer la nueva normativa sobre programación y contenido de la publicidad televisiva de alimentos de niños, prohibiéndose la publicidad de alimentos con alto contenido en grasa, sal y azúcar, en los canales de TV dirigidos a niños y niñas y el resto de los canales en programas dirigidos a menores entre 4 y 15 años (29).

La TV ha dado un vuelco en la época contemporánea en relación a sus funciones y usos, pasando de ser "una ventana abierta al mundo" a "una TV relacional"; de un medio pasivo, a un medio interactivo en el que el espectador manifiesta sus inquietudes y la TV se amolda a sus gustos y preferencias (30); por tanto, las intervenciones destinadas a mejorar la nutrición de los niños y niñas necesitan hacer frente a la variedad de factores sociales y físicos, incluyendo la TV; las cuales influyen en los patrones alimentarios de los menores (16). Su impacto debe considerarse dentro de la educación alimentaria-nutricional dirigida a sus cuidadores, de tal forma que haya coherencia en el discurso del equipo de salud con las prácticas que en el hogar se lleven a efecto.

\section{RESUMEN}

Antecedentes. La televisión es un medio de comunicación que desarrolla la cultura, entretenimiento, costumbres y hábitos en un ambiente sedentario, por lo que su consecuencia en la salud nutricional debe estudiarse. Objetivo. Correlacionar el tiempo destinado a ver TV con el estado nutricional y los hábitos alimentarios niños entre 3 y 6 años. Metodología. Estudio de corte transversal a 45 menores de edad residentes en la población Jardín del Este, en la ciudad de Talca (Chile), abordados mediante visita domiciliaria. Se evaluó el estado nutricional, aplicó una encuesta de tendencia de consumo y se registró el tiempo en ver televisión en casa/día. Los resultados se analizaron con Stata 10,1. Resultados. El 73,3\% de los niños ven televisión entre 4 y 7 horas diarias. El tiempo destinado para televisión fue de 3,5 $\pm 1,0$ horas en eutróficos, 4,7 \pm 0,9 horas en niños con sobrepeso y 5,6 $\pm 0,9$ horas en obesos $(p<0,001)$. Niños que consumen más porciones al día de azúcares pasan en promedio más horas de TV $(p<0,05)$. Conclusión. El tiempo destinado a la televisión en casa influye en el estado nutricional y en los hábitos alimentarios de los niños.

Palabras clave: televisión; estado nutricional; hábitos alimentarios; actividad física.

\section{BIBLIOGRAFÍA}

1. Moreno, T. La televisión, mediadora entre consumismo y obesidad. Rev Chil Nutr 2009; 36 (1): 46-52.

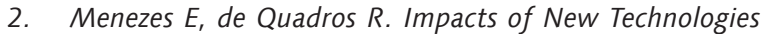
on Free-to-Air TV industry: Lessons from Selected Country Cases. J Technol Manag Innov 2009; 4 (4):82-93.

3. Santander P. Acceso y poder discursivo en las noticias de la televisión chilena. Rev Signos 2002; 35 (51-52):243-69.

4. Manios Y, Kourlaba G, Kondaki K, Grammatikaki E, Anastasiadou A y Roma-Giannikou E. Obesity and Television
Watching in Preschoolers in Greece: The GENESIS Study. Obesity 2009; 17, 2047-53.

5. Fernández $R$, Christofaro D, Casonato J, Costa rosa $R$, Costa F, Freitas I, Henrique Luiz Monteiro1, Ramos de Oliveira A. Leisure time behaviors: Prevalence, correlates and associations with overweight in Brazilian adults. A cross-sectional análisis Rev Med Chile 2010; 138: 29-35.

6. Castillo A, Kain J. Consejería en vida sana y cambio de conductas en escolares obesos: intervención controlada en madres/cuidadoras. Rev Chil Nutr 2010; 37 (2): 155-63.

7. Burdette H, Whitaker R, Kahn R, Harvey-Berino J. Association of maternal obesity and depressive symptoms with television-viewing time in low-income preschool children. Arch Pediatr Adolesc Med 2003; 157:894-9.

8. Rojas, V. (2008). Influencia de la televisión y videojuegos en el aprendizaje y conducta infanto-juvenil. Rev Chil Pediatr 2008; 79 (1): 80-5.

9. Crovetto M, Durán M, Guzmán M, Miranda C. Estudio descriptivo de la frecuencia y duración de la publicidad alimentaria emitida en la programación de canales de televisión asociados a Anatel. Rev Chil Nutr 2011; 38 (3): 290-9.

10. Amigo $H$, Bustos $P$, Erazo $M$, Cumsille $P$, Silva $C$. Factores determinantes del exceso de peso en escolares: Un estudio multinivel. Rev Méd Chile 2007; 135: 1510-8.

11. Ministerio de Salud, Chile. Encuesta Nacional de Salud 2009-2010. p. 17-19.

12. Quinteros Dolly S., Técnicas para la toma de medidas antropométricas. Centro de atención Nutricional. Segunda edición; Medellín; 1992 p. 5-32.

13. Barrera, G. Evaluación Nutricional del Crecimiento y del Riesgo Cardiovascular y Metabólico. Instituto de Nutrición y Tecnología y los Alimentos. Chile. 2010 p. 10-58.

14. Urteaga C, Pinheiro A. Investigación alimentaria: consideraciones prácticas para mejorar la confiabilidad de los datos. Rev Chil Nutr 2003; 30 (3): 235-42.

15. Olivares $S$, Zacarías I. Guía de alimentación saludable y necesidades nutricionales del adulto. Instituto de Nutrición y Tecnología de los Alimentos. Universidad de Chile. Disponible en: http://www.inta.cl/material_educativo/ cd/3GuiAli.pdf Consultado del 05 de septiembre del 2012.

16. Patrick $H$, Nicklas $T$. A review of family and social determinants of children's eating patterns and diet quality. J Am College Nutr 2005; 24 (2): 83-92.

17. Díaz X. et al. Análisis comparativo de la canasta básica de alimentos, pirámide alimentaria y recomendaciones nutricionales para preescolares y escolares chilenos: A comparative analysis. Rev Chil Pediatr 2006; 77(5): 466-72.

18. Loaiza S, Atalah E. Factores de riesgo de obesidad en escolares de primer año básico de Punta Arenas. Rev Chil Pediatr 2006; 77 (1): 20-6.

19. Peiró y Merma. Una mirada crítica a las repercusiones de la televisión en la educación. Polis, Rev Universidad Bolivariana 2011; 10 (29): 413-32.

20. García y Vargas. Conocimiento y percepciones culturales de los padres sobre los juguetes apropiados para sus hijos. Encuesta en dos Consultorios de Atención Primaria de Santiago Metropolitano. Rev Chil Pediatr 2005; 76 (4): 363-8.

21. Burdette H, Whitaker R, Kahn R, Harvey-Berino J. Association of maternal obesity and depressive symptoms with television-viewing time in low-income preschool children. Arch Pediatr Adolesc Med 2003; 157: 894-9.

22. Manios Y, Kondaki K, Kourlaba G, Grammatikaki E, Birbilis $M$ and loannou E. Television viewing and food habits in 
toddlers and preschoolers in Greece: the GENESIS study. European J Pediatr 2009; 168 (7): 801-8.

23. Vergara del S. A, Chávez $P$, Vergara E. Televidencia y vida cotidiana de la infancia. Un estudio de casos con niños y niñas de Santiago. Polis. Rev Universidad Bolivariana 2010; 9 (26): 371-96.

24. Harris J, Bargh J. The Relationship between Television Viewing and unhealthy eating: implications for children and media interventions. Health Commun 2009; 24(7): 660-73.

25. Bankoski A, Kathryn H. Jacobsen K, Pawloski L, Burley J, Gaffney $K$, Jaimovich S, Campos C. Relación entre prácticas alimentarias reportadas por niños chilenos y preferencias de los padres para su consumo. Rev Chil Nutr 2010; 37 (3): 352-8.

26. Pantic, Igor et al. Screen viewing, body mass index, cigarette smoking and sleep duration in Belgrade University student population: results of an observational, crosssectional study. Rev Méd Chile 2011; 139 (7): 896-901.

27. Coon KA, Tucker KL. Television and children's consumption patterns. A review of the literature. Minerva Pediatr 2002; 54(5):423-36.

28. Guran T, Bereket A. International epidemic of childhood obesity and television viewing. Minerva Pediatr 2011; 63(6):483-90.

29. Adams J, Tyrrell R, Adamson AJ, White M Effect of restrictions on television food advertising to children on exposure to advertisements for 'Less Healthy' Foods: Repeat Cross-Sectional Study. PLOS ONE; 2012 7(2): Disponible en http://www.plosone.org/article/ info\%3Adoi\%2F10.1371\%2Fjournal.pone.0031578. Acceso el 10 de marzo de 2012.

30. Fuentes, Ivonne. La argumentación y las emociones en el debate televisivo. Rev Signos 2009; 42 (70): 171-95. 